patients who are dying/providing bereavement support (65\% confident).

Conclusions Tests of changes need to be small enough to try within existing workloads. The focus for July to Sept 2018 (cycles: 1 clinician, 10 patients informed by patient/relative experience) is:

- improved handover and future care planning on discharge (acute frailty)

- earlier recognition, communication alongside improving knowledge/access to SPC (A\&E).

\section{P-201 TRANSFORMATION OF WARD COMPANIONS}

Suzann Chantrill, Minaxi Patel. LOROS Hospice, Leicester, UK

\subsection{6/bmjspcare-2018-hospiceabs.226}

Background LOROS decided to develop the scope of volunteering roles within the inpatient unit to allow volunteers to become 'Ward Companions'. This change was implemented following patient feedback that suggested greater volunteer involvement would be beneficial to patients' experience of hospice care.

Aims Ward companions aim to enhance the patient's stay by providing practical and emotional support and importantly, a sense of normality. Companions engage with patients in many ways; by playing games, through reading, assisting at meal times, or just 'being there' as a reassuring presence in times of need. Depending on the volunteer's own qualifications and interests, they may also provide assistance with patient care, hairdressing, nail and beauty services. Companions have also supported patients to draft their last wishes and create memory boxes for loved ones. Companions have supported patients that do not have family or friends.

Methods The Clinical Volunteer Coordinator has been instrumental in devising role descriptions and encouraging volunteers to contribute their skills in this area. All volunteers are required to complete a thorough training programme to ensure they are sufficiently equipped to support patients. All volunteers report to a ward team leader who briefs them on individual patient requirements and requests.

Conclusion There are currently 32 volunteers enrolled as ward companions for LOROS and their contribution to inpatients has been invaluable, as evidenced by numerous 'thank you' cards and LOROS 'Have Your Say' feedback cards. The Ward Companions initiative has been shared via BBC News and Facebook, highlighting the impact the role has had and the potential for hospices to adopt this approach in the future. It's 'the little things' in life that often mean so much and the attention, care, compassion, trust and empathy afforded by volunteer ward companions has significantly improved patients' experience of LOROS.

\section{P-202 CO-PRODUCTION IN PALLIATIVE CARE}

Trudi Leighton. North London Hospice, London, UK

10.1136/bmjspcare-2018-hospiceabs.227

Background The Health and Wellbeing Centre at North London Hospice has undergone significant change in its service provision. A priority for improvement project for the organisation was to develop a co-production model for new service developments.

Aims Establish within our needs stratified model one-to-one complex interventions, shared professional care and community/social engagement using a co- production model; develop a means of designing and delivering new interventions; empower users and staff to develop self-management skills; 'doing with, not doing to'; provide a resource for the organisation.

Methods A steering group provided support, guidance and oversight during the project. A co-production framework was adapted. The needs stratified model provided the framework for the level of intervention. User engagement as an equal partner in planning, delivering and assessing their care.

Example Photography 'Catching the Light. Users expressed an interest in using photography as a medium for expression and enjoyment. An exhibition of a patient's photography launched the initial co-production meeting, facilitated by a staff member. All stakeholders including camera societies were invited. (lived experience). Examples of using photography in palliative care was researched (research literature). Formulation of a plan at the exhibition, including recruiting volunteers with photographic technical ability to support the group (knowledge and expertise).The relationship between users and staff was equal, open and honest and led to a creative, supportive and powerful group who were empowered (candour, accountability, respect, communication skills).

Conclusion A collaborative co-produced model enabled our service users, local community and staff to be empowered to design, plan and deliver services of relevance to their needs. The culture of the service shifted; ownership, openness and honesty, sharing decision making, respect and valuing our users as equals grew and led the organisation to examine the relationship with users.

\section{P-203 A RECIPE FOR SUCCESS: CO PRODUCING A USER FORUM}

Giselle Martin-Dominguez, Deborah Mosdall. North London Hospice, London, UK

\subsection{6/bmispcare-2018-hospiceabs.228}

Which recipe? Despite the use of focus groups at our hospice there had never been a user forum due to concerns that users might be too unwell or too upset to participate regularly.

Shopping for the right ingredients The project aim was to set up a Forum where service users could provide feedback on concerns and also enable the hospice to hear views on plans to develop services.

Following the recipe Current users from our outpatient service where patients are generally fitter than those from other services were targeted as the initial focus for developing a user forum. Two patients joined a project steering group and an initial meeting was held with patients, staff and volunteers to co-produce the structure of the User Forum (which patients prefer to be called 'Feedback Group') and how to promote attendance.

The perfect cake Eleven groups have taken place. Topics discussed have included catering, our annual quality projects, our three-year strategic plan and user submission to the All-Party Parliamentary Group on Improving Access to Hospices. As a result a user is now a member of our Improving Access Project steering group, users are co-producing two other quality projects, have re-named our outpatient service as well as influenced a change to the consistency of our soups and 